\title{
Transient analysis of spectrally asymmetric magnetic photonic crystals with ferromagnetic losses
}

\author{
K.-Y. Jung, B. Donderici, and F. L. Teixeira \\ ElectroScience Laboratory and Department of Electrical and Computer Engineering, The Ohio State University, 1320 Kinnear Road, \\ Columbus, Ohio 43212, USA \\ (Received 6 April 2006; revised manuscript received 25 August 2006; published 23 October 2006)
}

\begin{abstract}
We analyze transient electromagnetic pulse propagation in spectrally asymmetric magnetic photonic crystals (MPCs) with ferromagnetic losses. MPCs are dispersion-engineered materials consisting of a periodic arrangement of misaligned anisotropic dielectric and ferromagnetic layers that exhibit a stationary inflection point in the (asymmetric) dispersion diagram and unidirectional frozen modes. The analysis is performed via a late-time stable finite-difference time-domain method (FDTD) implemented with perfectly matched layer (PML) absorbing boundary conditions, and extended to handle (simultaneously) dispersive and anisotropic media. The proposed PML-FDTD algorithm is based on a D-H and B-E combined field approach that naturally decouples the FDTD update into two steps, one involving the (anisotropic and dispersive) constitutive material tensors and the other involving Maxwell's equations in a complex coordinate space (to incorporate the PML). For ferromagnetic layers, a fully dispersive modeling of the permeability tensor is implemented to include magnetic losses in a consistent fashion. The numerical results illustrate some striking properties of MPCs, such as wave slowdown (frozen modes), amplitude increase (pulse compression), and unidirectional characteristics. The numerical model is also used to investigate the sensitivity of the MPC response against excitation (frequency and bandwidth), material (ferromagnetic losses), and geometric (layer misalignment and thickness) parameter variations.
\end{abstract}

DOI: 10.1103/PhysRevB.74.165207

PACS number(s): 78.20.Bh, 41.20.Jb, 78.20.Ls, 42.65.-k

\section{INTRODUCTION}

Spectrally asymmetric magnetic photonic crystals (MPCs) are dispersion-engineered periodic materials that have been recently proposed to yield unique properties such as wave slowdown ("frozen modes"), amplitude increase (via pulse compression), and electromagnetic (EM) unidirectionality at microwave frequencies. ${ }^{1-3}$ Periodic stacks composed of misaligned anisotropic dielectric layers ( $A$ layers) and ferromagnetic layers ( $F$ layers) with a proper choice of geometry and tensor parameters display an asymmetric dispersion relation $\omega(k)$ with a stationary inflection point (SIP) in a forward direction (for example, from left to right) and no SIP in a backward direction (for example, from right to left). ${ }^{1}$ Since group velocities are extremely low near the SIP, EM pulses seem to be "frozen" inside MPCs when propagating in the forward direction. At the same time, these forward propagating EM pulses can exhibit a dramatic growth in amplitude inside the MPCs. In the backward direction, EM waves inside the MPCs propagate in an ordinary fashion. ${ }^{2}$ Although MPCs were first proposed for (semi)-infinite periodic structures, finite-size MPCs also display similar properties and thus support frozen modes. ${ }^{4}$

The finite-difference time-domain (FDTD) method is particularly suited for obtaining the transient response of complex materials including bulk media with anisotropic and dispersive responses. ${ }^{5-9}$ However, two main challenges exist for FDTD analysis of MPCs: (i) accurate treatment of both anisotropic dielectric and ferromagnetic bulk responses simultaneously, and (ii) late-time time-domain stability to simulate extremely slow-moving EM pulses. In addition, because frozen modes exist in the very narrow bandwidth, a narrowband incident pulse should be employed in FDTD simulations. These two features - a low group velocity and a narrowband pulse-require accurate late-time FDTD results. ${ }^{10,11}$

In this work, we perform a transient analysis of electromagnetic pulse propagation in spectrally asymmetric MPCs. The analysis is done by means of an extended FDTD algorithm with perfectly matched layers (PML-FDTD) for general anisotropic dispersive linear media. The PML-FDTD algorithm developed here extends D-H and B-E PML formulations developed in Refs. 12 and 13. The motivation behind using D-H and B-E PML formulations stems from the fact that the equations involving the (anisotropic and dispersive) material parameters are naturally decoupled from the PML-FDTD equations involving finite-difference approximations to spatial derivatives in a complex coordinate space. ${ }^{14,15}$ For this reason, this class of PML implementation is also often referred to as material-independent PML.

FDTD equations for nondispersive anisotropic dielectric media can be formulated without much difficulty. However, FDTD equations for lossy ferromagnetic media should be carefully formulated. In the past, two basic approaches have been reported to model ferromagnetic media in the FDTD. The first approach is based upon the equation of motion of the magnetization. ${ }^{16}$ In this approach, the Yee FDTD staggered mesh should be modified. The second approach uses frequency-dependent permeability tensors where the resulting FDTD update equations are obtained by recursive convolution $^{17,18}(\mathrm{RC})$ to account for frequency dispersion. In order to be combined with D-H and B-E PML formulations, the latter approach is preferred for ferromagnetic media. However, our extensive numerical experiments have indicated that the RC approach suffers from late-time instabilities in this case. Because of this, a PML-FDTD formulation for ferromagnetic media is derived here directly from the permeability tensors without recursive convolutions. This formulation is a generalization (to anisotropic dispersive media) of the auxiliary differential equation (ADE) approach 

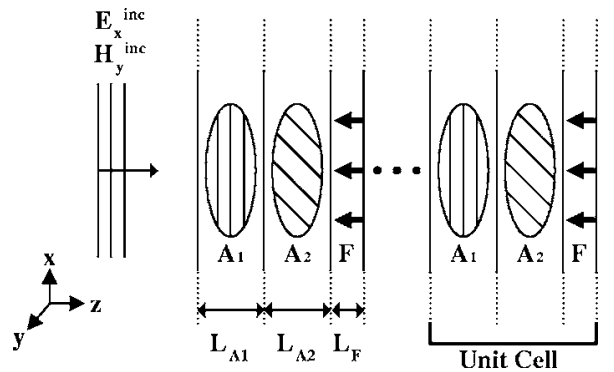

FIG. 1. Spectrally asymmetric magnetic photonic crystal with unit cell composed of two (misaligned) anisotropic dielectric layers and one ferromagnetic layer. The MPC is surrounded by air and is under a plane wave excitation. Also shown is the direction of the dc biasing magnetic field for the $F$ (ferrite) layers.

previously developed for isotropic dispersive media. ${ }^{19}$

The remainder of this paper is organized as follows. Basic properties of MPCs are reviewed in Sec. II. In Sec. III, numerical results illustrate some of the MPC's striking properties, such as wave slowdown, amplitude increase, and unidirectionality. In Sec. IV, we investigate the sensitivity of the MPC electromagnetic response against excitation (frequency and bandwidth), material (ferromagnetic losses), and geometric (layer misalignment and thickness) parameter variations. Concluding remarks are provided in Sec. V. Details on the PML-FDTD algorithm are included in the Appendix.

\section{SPECTRALLY ASYMMETRIC MPC}

The simplest way to achieve a MPC is through a periodic structure whose unit cell is composed of two $A$ layers (misaligned anisotropic dielectrics) with different orientations and one $F$ layer (ferrite), ${ }^{1}$ as depicted in Fig. 1. Spectral asymmetry is related to time reversal asymmetry and space inversion asymmetry. Magnetically polarized media must be used to achieve time reversal asymmetry and at least three layers in a unit cell must be employed to achieve space in- version asymmetry. ${ }^{1}$ Figure 2 shows a $\omega-k$ band diagram for a MPC, where the asymmetry between positive and negative Bloch wavenumbers is evident, and the slope of the dispersion curve (and hence the group velocity) near the SIP is extremely small.

Because frozen modes in MPCs exist in a very narrow bandwidth, it is often not of fundamental importance to consider dispersion in $A$ layers. However, off-diagonal terms in the permeability tensor model of lossless $F$ layers exhibit a $90^{\circ}$ phase difference from the diagonal terms [see Eq. (3)]. Moreover, it is also important to study the effect of ferromagnetic losses. Hence, it is highly desirable to model $F$ layers consistently by considering full dispersive models.

We assume an $e^{j \omega t}$ time convention in what follows. The constitutive tensor of $A$ layers with anisotropy in the $x y$ plane can be expressed as ${ }^{1}$

$$
\begin{aligned}
\overline{\overline{\epsilon_{A}}}(\omega) & =\epsilon_{0}\left[\begin{array}{ccc}
\epsilon_{x x} & \epsilon_{x y} & 0 \\
\epsilon_{x y} & \epsilon_{y y} & 0 \\
0 & 0 & \epsilon_{z z}
\end{array}\right] \\
& =\epsilon_{0}\left[\begin{array}{ccc}
\epsilon_{A}+\delta_{A} \cos \left(2 \varphi_{A}\right) & \delta_{A} \sin \left(2 \varphi_{A}\right) & 0 \\
\delta_{A} \sin \left(2 \varphi_{A}\right) & \epsilon_{A}-\delta_{A} \cos \left(2 \varphi_{A}\right) & 0 \\
0 & 0 & \epsilon_{z z}
\end{array}\right],
\end{aligned}
$$

$$
\overline{\overline{\mu_{A}}}(\omega)=\mu_{0} \mu_{r} \overline{\bar{I}},
$$

where $\overline{\bar{I}}$ is the identity matrix. The parameter $\delta_{A}$ is the magnitude of in-plane anisotropy, and $\varphi_{A}$ is the orientation angle of the principal axis of the permittivity tensor in the $x y$ plane.

For a saturated ferrite with a $z$-directed dc biasing magnetic field, the constitutive tensor is expressed as ${ }^{20}$

$$
\overline{\overline{\epsilon_{F}}}(\omega)=\epsilon_{0} \epsilon_{r} \overline{\bar{I}},
$$

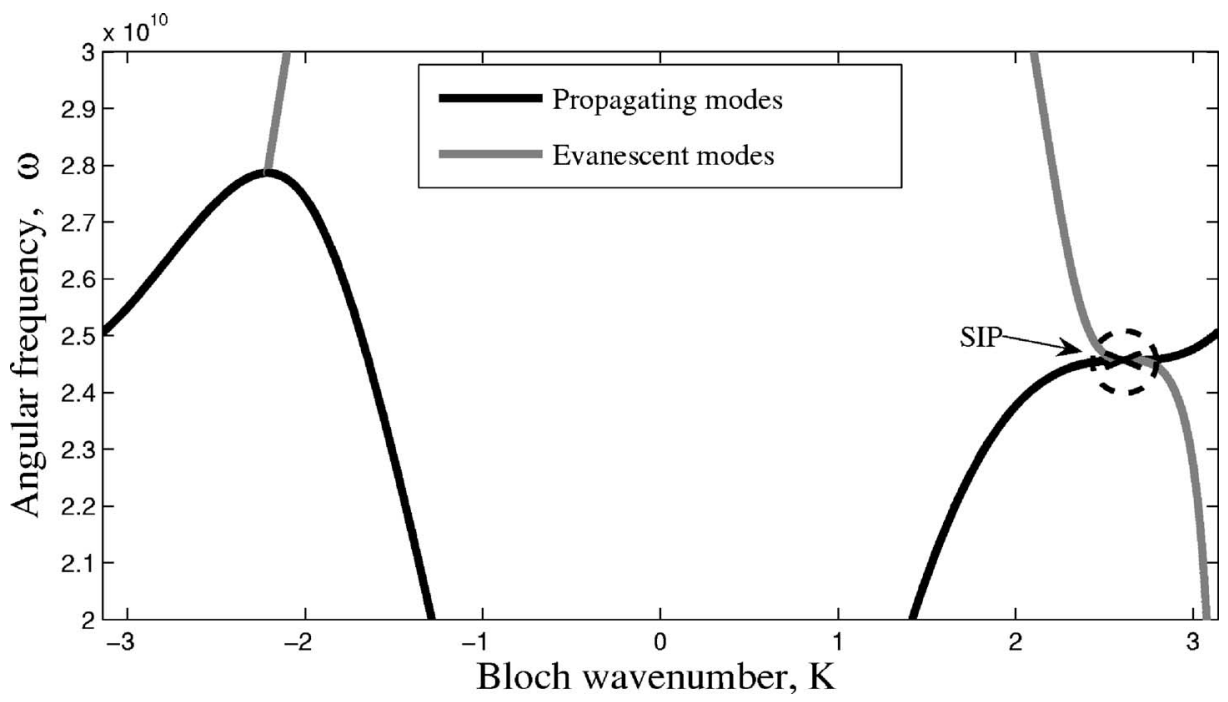

FIG. 2. Band diagram of the MPC of Fig. 1, showing a stationary inflection point for positive wave number, and the asymmetry of the diagram for positive and negative wave number (corresponding to forward and backward propagation, respectively). 


$$
\overline{\overline{\mu_{F}}}(\omega)=\mu_{0}\left[\begin{array}{ccc}
1+\chi_{x x}(\omega) & \chi_{x y}(\omega) & 0 \\
\chi_{y x}(\omega) & 1+\chi_{y y}(\omega) & 0 \\
0 & 0 & 1
\end{array}\right] \text {, }
$$

where

$$
\begin{gathered}
\chi_{x x}(\omega)=\chi_{y y}(\omega)=\frac{\left(\omega_{0}+j \omega \alpha\right) \omega_{m}}{\left(\omega_{0}+j \omega \alpha\right)^{2}+(j \omega)^{2}}, \\
\chi_{x y}(\omega)=-\chi_{y x}(\omega)=\frac{j \omega \omega_{m}}{\left(\omega_{0}+j \omega \alpha\right)^{2}+(j \omega)^{2}} .
\end{gathered}
$$

In the above, $\alpha$ is the damping (loss) constant, $\omega_{0}=\gamma H_{0}$, and $\omega_{m}=\gamma 4 \pi M_{s}$, where $\gamma$ is the gyromagnetic ratio, $H_{0}$ is the dc biasing magnetic field magnitude, and $M_{s}$ is the dc saturation magnetization. References 1 and 2 discuss in detail the electromagnetic mechanisms underlying the MPC behavior.

\section{MPC PROPERTIES}

We use the PML-FDTD formulation described in the Appendix. As depicted in Fig. 1, the dc biasing magnetic field is $-z$ directed in the $F$ layers. The geometrical dimensions are as follows: $L_{A 1}=L_{A 2}=5 \mathrm{~mm}$ and $L_{F}=1 \mathrm{~mm}$. For $A$ layers, the constitutive tensor parameters are $\epsilon_{A 1}=\epsilon_{A 2}=7 ; \delta_{A 1}=\delta_{A 2}=6$; $\varphi_{A 1}=0^{\circ}, \varphi_{A 2}=36.0963^{\circ} ; \epsilon_{z z, A 1}=\epsilon_{z z, A 2}=1 ; \mu_{r, A 1}=\mu_{r, A 2}=1$. For $F$ layers, the constitutive tensor parameters are given by $\epsilon_{r}$ $=5 ; \quad \omega_{0}=36.503 \times 10^{9} \mathrm{rad} / \mathrm{s} ; \omega_{m}=73.006 \times 10^{9} \mathrm{rad} / \mathrm{s}$. The total-field/scattered-field (TF/SF) formulation was used as a source condition. ${ }^{19}$ The excitation pulse $\left(E_{x}\right)$ is a sine wave modulated by a Gaussian pulse with unit peak amplitude [the operating angular frequency is $24.565 \times 10^{9} \mathrm{rad} / \mathrm{s}$ and the $5 \%$ fractional bandwidth (BW) is $0.01 \%$ ]. A space step $\Delta z$ $=0.08333 \mathrm{~mm}$ and Courant factor of 0.98 were used. All simulations were performed on a vector processor Cray X1 supercomputer.

Figure 3 shows snapshots of $\left|E_{x}\right|$ in the presence of a lossless MPC with 500 unit cells for the forward propagating pulse. The results show that the peak value of the electric field increases by about eight times inside this MPC. While the incident pulse spans about $10000 \lambda_{0}$ in air, the spatial span of the pulse inside the MPC is extremely narrowed. In other words, when the incident pulse enters the MPC in the forward direction, the EM wave is drastically slowed down ("frozen"), the pulse is compressed, and the amplitude increases dramatically. However, because the (relative) group velocity changes drastically near a SIP, the pulse propagation is highly dispersive, as can be seen from the pulse spread in Fig. 3.

To illustrate the unidirectional property of MPCs, the spatial order of layers is reversed (F-A2-A1 layer arrangement) with respect to the previous one ("backward" propagation). Figure 4 shows snapshots of $\left|E_{x}\right|$ in the presence of this lossless MPC. The pulse propagates at much faster speed in this case, and no growth in amplitude is observed in this backward propagation. As seen from Fig. 4, the backward propagating pulse reaches the far end of the MPC at a much earlier time, and then it is reflected toward the near end of the MPC. The direction of reflected pulse is now forward and hence the

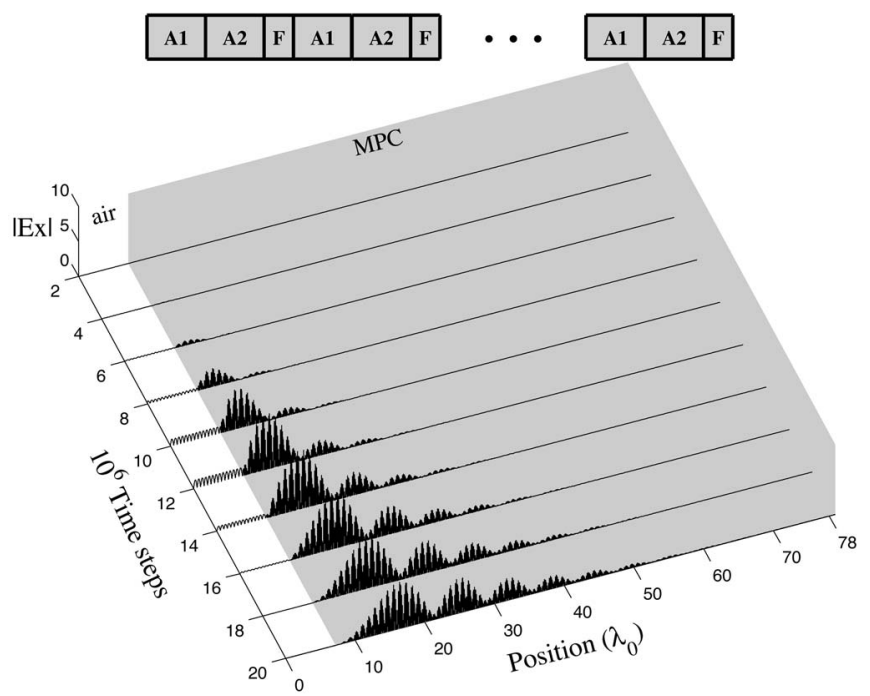

FIG. 3. Snapshots of $\left|E_{x}\right|$ due to a narrowband, unit-amplitude pulse incident on a lossless MPC along the forward MPC direction. The $5 \%$ fractional bandwidth of the incident pulse is equal to $0.01 \%$, with $\Delta t=0.272 \mathrm{ps}$ and $\lambda_{0}=76.733 \mathrm{~mm}$. The incident pulse has unit amplitude, and the results clearly show the resulting field amplification inside the MPCs.

reflected pulse shows the amplitude increase and wave slowdown.

Because the group velocity for a finite bandwidth pulse including the SIP is spread around zero value, it is not meaningful to try to determine an effective or average group ve-

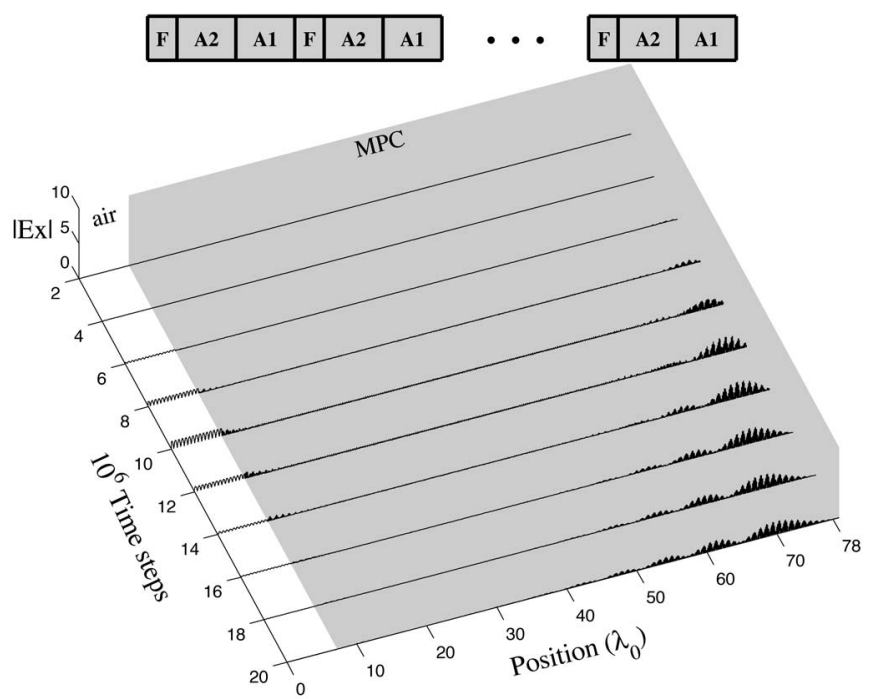

FIG. 4. Snapshots of $\left|E_{x}\right|$ due to a narrowband, unit-amplitude pulse incident on a lossless MPC along the backward MPC direction (i.e., the MPC is reversed with respect to the previous figure). The BW of the incident pulse is again $0.01 \%$, with $\Delta t=0.272 \mathrm{ps,}$ and $\lambda_{0}=76.733 \mathrm{~mm}$. In this figure, the pulses visible at the fourth and later snapshots are produced by reflection at the far end of the MPC. The (backward) propagating pulse reaches the far end of the MPC at very early times (having propagated at ordinary group velocity) and is reflected toward the near end of the MPC along the forward direction with a much slower group velocity. 


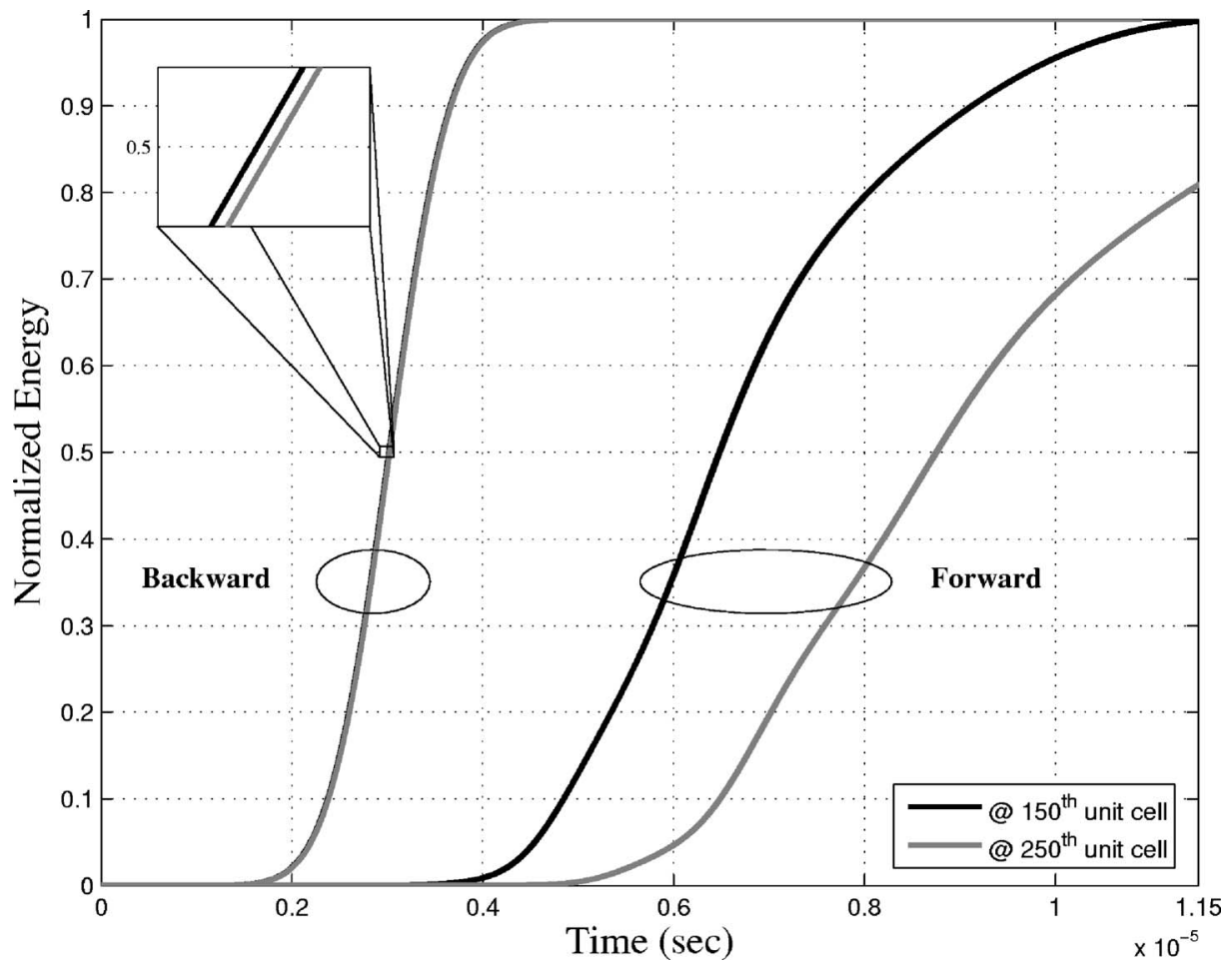

FIG. 5. Normalized energy to calculate a (half-time, non-characteristic) effective rate of energy transport. This is obtained by dividing the distance between two observation points by the time delay necessary for half of the pulse energy to pass through the two observation points. The inset diagram $(100 \times$ zoom window $)$ is included to illustrate the much shorter time delay for backward propagation between the same two observation points.

locity for the pulse in this highly dispersive regime. Instead, we adopt here the approach described in Ref. 21 to obtain an "effective rate" of (half-time) energy transport. This is determined by calculating the times at which half of the electromagnetic energy has been collected at two observation points (note that this is position dependent and not a characteristic quantity of the MPC). To avoid reflections at the interface of the MPC and air at the far end of the MPC, 2000 unit cells are employed. Figure 5 shows the instantaneous (integrated) collected energy, which is normalized at the center of the 150th unit cell. By dividing the distance between the two observation points $(\Delta z=1.1 \mathrm{~m})$ by the difference of times to collect half of the energy at these two points $(\Delta t$ $=2.274 \mu \mathrm{s}$ ), the effective rate of energy transport for the forward propagating pulse is found to be about $0.0016 c_{0}$. A similar procedure is carried out for the backward direction. The time difference to collect half the energy at the two observation points is now $\Delta t=9 \mathrm{ps}$, which implies an effective rate of energy transport equal to $0.4074 c_{0}$. Hence, the effective half-time energy transfer slowdown is equal to about 250 times.

\section{SENSITIVITY ANALYSIS}

Next, we use this PML-FDTD formulation to illustrate the sensitivity of the MPC response for five different scenarios: (i) a lossless MPC with frequency-mismatched incident pulses, (ii) a lossless MPC with wider band incident pulses, (iii) a lossy MPC with different number of unit cells, (iv) a lossless MPC with random variations on $A$ layer misalignments, and (v) a lossless MPC with random variations on $A$ and $F$ layer thicknesses.

\section{A. Frequency sensitivity}

To illustrate the impact of frequency deviations on the pulse propagation inside the MPC, we change the center fre-

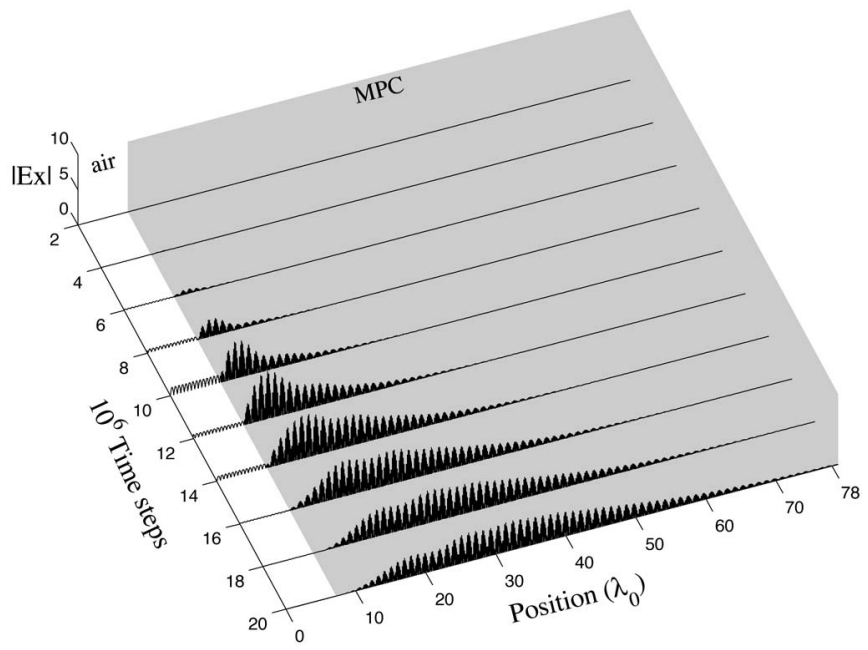

FIG. 6. Snapshots of $\left|E_{x}\right|$ in a lossless MPC. The narrowband, unit-amplitude incident pulse is shifted in frequency, with $\delta \omega$ $=0.01 \%$. In this simulation, $\Delta t=0.272 \mathrm{ps}$ and $\lambda_{0}=76.733 \mathrm{~mm}$. Note the increase in the effective group velocity and the decrease in $\left|E_{x}\right|$ compared to Fig. 3. 


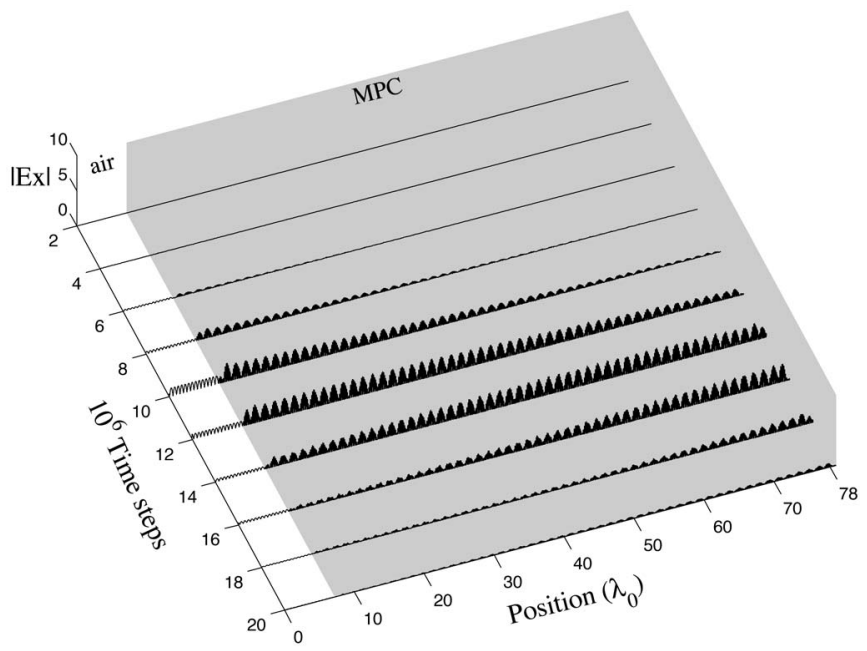

FIG. 7. Snapshots of $\left|E_{x}\right|$ in a lossless MPC. The narrowband, unit-amplitude incident pulse is shifted in frequency, with $\delta \omega$ $=0.1 \%$. In this simulation, $\Delta t=0.272 \mathrm{ps}$ and $\lambda_{0}=76.733 \mathrm{~mm}$. Note the further increase in the effective group velocity and the decrease in $\left|E_{x}\right|$ compared to Figs. 3 and 6 .

quency of the incident pulse from $\delta \omega=0.01 \%$ and $\delta \omega$ $=0.1 \%$. Figure 6 shows snapshots of $\left|E_{x}\right|$ in the presence of the lossless MPC for the frequency-shifted incident pulse with $\delta \omega=0.01 \%$. The peak value of $\left|E_{x}\right|$ inside the MPC is decreased, and the average group velocity is increased, as compared to the matched-frequency case (Fig. 3). Figure 7 shows snapshots of $\left|E_{x}\right|$ in the presence of the lossless MPC for the frequency-shifted incident pulse with $\delta \omega=0.1 \%$. As expected, the increase in the group velocity and the decrease in $\left|E_{x}\right|$ are considerably more pronounced with a larger frequency mismatch.

It is also interesting to examine the sensitivity to the pulse central frequency versus the slope of the dispersion curve. It is expected that the MPC response becomes less sensitive to the central frequency for a (quasi-)MPC, where the slope of the dispersion curve (and hence the group velocity) becomes small but not zero (i.e., no true SIP is present). Figure 8 shows dispersion relations for the original MPC and two modified quasi-MPC designs where group velocities are very small but not zero over a frequency range near the SIP frequency. These new quasi-MPC designs are obtained by changing the thickness of the $F$ layer (cf. Fig. 8). Snapshots of $\left|E_{x}\right|$ at time step $12 \times 10^{6}$ are shown in Fig. 9 for the MPC with $L_{F}=1.0833 \mathrm{~mm}$ and Fig. 10 for the MPC with $L_{F}$ $=1.1667 \mathrm{~mm}$. Compared to the results shown in Figs. 3, 6, and 7 , although the amplitude growth and pulse compression effects are diminished as expected, the frequency sensitivity becomes less critical for the quasi-MPC designs. Table I provides a comparison for a quantitative measure of the frequency sensitivity against the slope of dispersion curve. This measure is defined by the normalized difference between the $\left|E_{x}\right|$ envelopes, $\mathcal{E}_{x}$ for the matched-frequency $(\delta \omega=0)$ and frequency-shifted cases $(\delta \omega \neq 0)$, and integrated along the MPC length:

$$
S_{F}=\int d z \frac{\mathcal{E}_{x}(z, \delta \omega \neq 0)-\mathcal{E}_{x}(z, \delta \omega=0)}{\mathcal{E}_{x}(z, \delta \omega=0)} .
$$

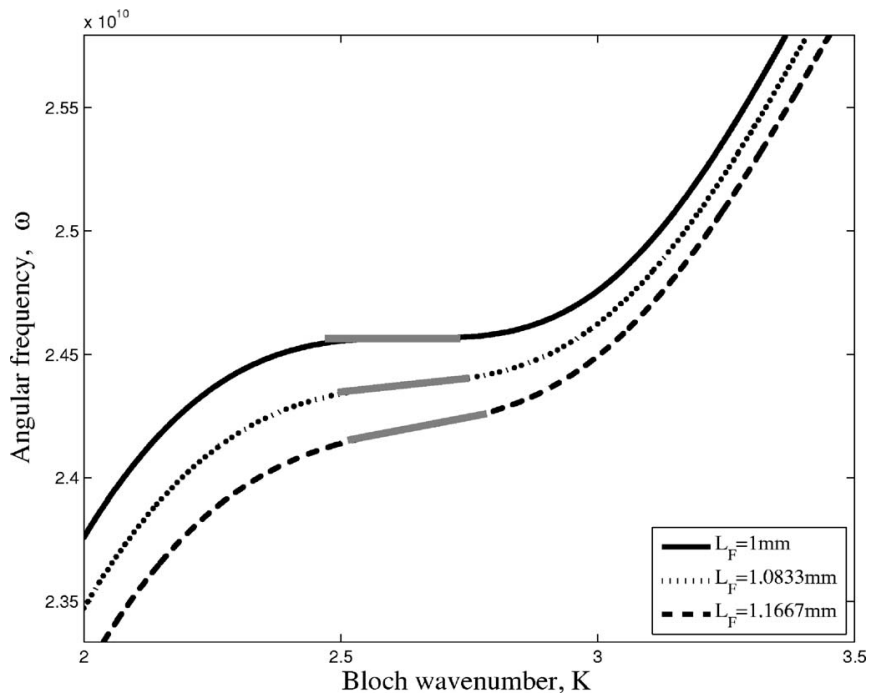

FIG. 8. Dispersion curves of spectrally asymmetric MPCs with zero group velocity (SIP) and nonzero (but small) group velocities, with nearly linear slopes around the SIP frequency. The solid curve corresponds to the original MPC (Fig. 2). The dashed and dotted curves indicate the modified (quasi-)MPCs. Also illustrated is the group velocity at central frequency for each case by the slope of the gray lines.

\section{B. Wideband pulse response}

In order to compare the relative wideband pulse response against the narrowband response, we change the $\mathrm{BW}$ of the excitation pulse from $0.01 \%$ to $0.1 \%$. Figure 11 shows snapshots of $\left|E_{x}\right|$ in the presence of the lossless MPC for a BW of $0.1 \%$. The peak field amplitude is reduced compared to the $0.01 \%$ case. Because the group velocity in the frequency range for a BW of $0.1 \%$ near the SIP is considerably spread out, dispersion is increased, as seen from Fig. 11. The "effective" group velocity is also increased compared to the $0.01 \%$ case, as expected.

\section{Ferromagnetic losses}

Next, we assess the sensitivity of the MPC response to different ferromagnetic loss coefficients $\alpha$ [cf. Eq. (3)]. The EM pulse (not shown here) inside a MPC with $\alpha=10^{-5}$ is nearly identical to that in the lossless MPC case $(\alpha=0)$, with relative $\left|E_{x}\right|$ peak amplitude ratio equal to 0.982 . Figure 12 shows snapshots of $\left|E_{x}\right|$ for a MPC with $\alpha=10^{-4}$. The decrease in the pulse amplification, compared to the lossless case in Fig. 3, is apparent here. The peak $\left|E_{x}\right|$ ratio of this lossy MPC to the lossless MPC is 0.868. Furthermore, since magnetic loss effects are cumulative, the EM pulse amplitude is more strongly suppressed as it penetrates more deeply into the crystal. Note, however, that such ferromagnetic losses do not destroy the essential properties (wave slowdown and pulse compression) of the MPC response.

We also evaluate the interplay of ferromagnetic losses and the total number of unit cells in the MPC response. Figure 13 plots the peak amplitude increase in a lossy MPC with different numbers of unit cells. As expected, a sufficient number of unit cells and a small ferromagnetic loss coefficient must 

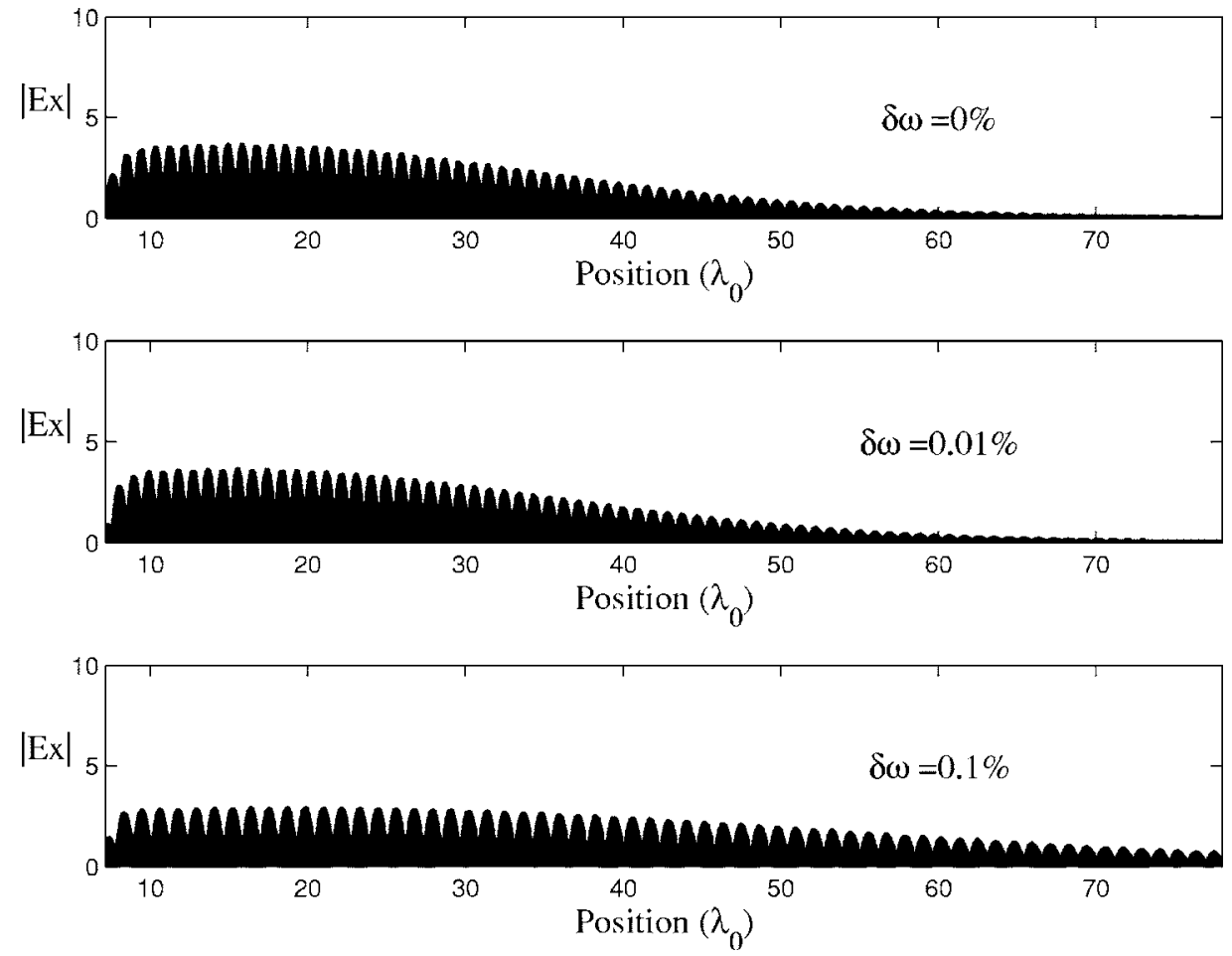

FIG. 9. Snapshots of $\left|E_{x}\right|$ inside the MPC at time step $12 \times 10^{6}$, with $L_{F}=1.0833 \mathrm{~mm}$. In this case, $\Delta t=0.272 \mathrm{ps}$ and $\lambda_{0}=76.733 \mathrm{~mm}$. The top plot indicates the matched-frequency case $\delta \omega=0 \%$, the center plot indicates the frequency-shifted case $\delta \omega=0.01 \%$, and the bottom plot indicates the frequency-shifted case $\delta \omega=0.1 \%$. Note the reduced frequency sensitivity in $\left|E_{x}\right|$ compared to the responses shown in Figs. 3 , 6 , and 7.
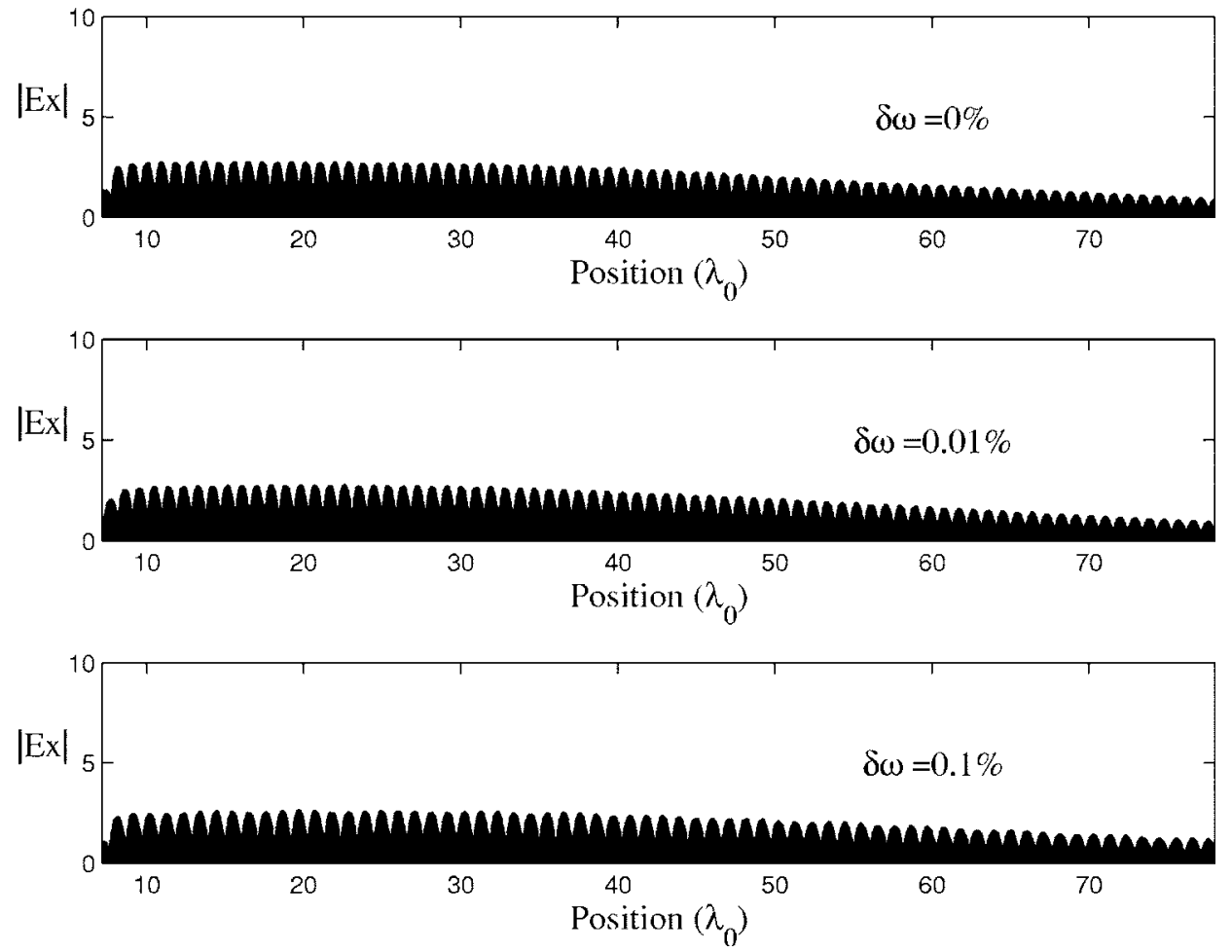

FIG. 10. Same as Fig. 9, now with $L_{F}=1.1667 \mathrm{~mm}$. Note the further reduced frequency sensitivity, compared to Fig. 9. 
TABLE I. Comparison of frequency sensitivities $S_{F}$.

\begin{tabular}{lcc}
\hline \hline MPC & $S_{F}(\delta \omega=0.01 \%)$ & $S_{F}(\delta \omega=0.1 \%)$ \\
\hline$L_{F}=1 \mathrm{~mm}^{\mathrm{a}}$ & 0.782 & 2.561 \\
$L_{F}=1.0833 \mathrm{~mm}$ & 0.448 & 0.710 \\
$L_{F}=1.1667 \mathrm{~mm}$ & 0.445 & 0.456 \\
\hline \hline
\end{tabular}

${ }^{\mathrm{a} O r i g i n a l ~ M P C}$ with true SIP.

be present to produce higher amplitudes. It can also be seen that the relative $\left|E_{x}\right|$ peak amplitude ratio is nearly constant for MPCs with more than 100 unit cells.

\section{Imperfect $\boldsymbol{A}$ layer misalignment}

To illustrate the effects of orientation misalignments of the $A$ layers, we incorporate random deviations in the orientation angles of the $A$ layers. In this case, $\varphi_{A 1}$ and $\varphi_{A 2}$ are described by Gaussian random variables $\varphi_{A 1}=0^{\circ}+N\left(0, \zeta_{\phi}^{2}\right)$, $\varphi_{A 2}=36.0963^{\circ}+N\left(0, \zeta_{\phi}^{2}\right)$. Four different standard deviations $\left(\zeta_{\phi}=1.5^{\circ}, 3^{\circ}, 4.5^{\circ}, 6^{\circ}\right)$ are considered. For each distribution, an ensemble with 15 realizations is used.

The solid line in Fig. 14 interpolates the (ensembleaveraged) peak value of $\left|E_{x}\right|$ inside lossless MPCs for the various $\zeta_{\phi}$. A narrowband incident pulse is used for these results. For reference, the peak $\left|E_{x}\right|$ for the frequency-shifted (narrowband) pulse with $\delta \omega=0.01 \%$ and $\delta \omega=0.1 \%$ and the wider band pulse with a BW of $0.1 \%$ are also displayed at $\zeta_{\phi}=0^{\circ}$. As seen from this figure, small deviations in the misalignment angle can have a strong impact on the MPC response.

\section{E. Imperfect layer thickness}

To elucidate the effects of variations on the layer thickness, we include (Gaussian distributed) random deviations in the thicknesses of $A$ and $F$ layers. Three different standard

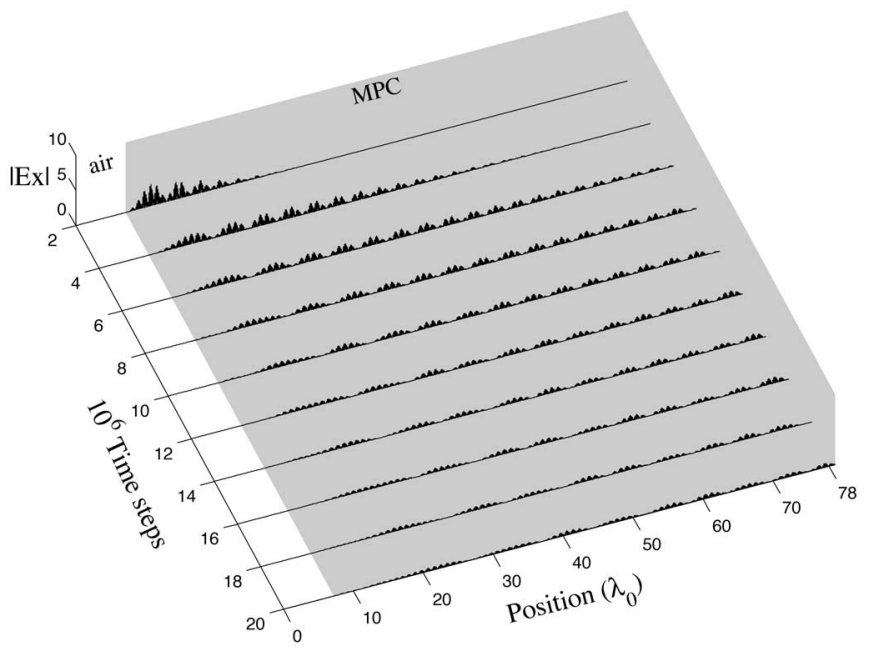

FIG. 11. Snapshots of $\left|E_{x}\right|$ in lossless MPC (incident pulse of unit amplitude with wider frequency band, $\mathrm{BW}=0.1 \%$ ). In this case, $\Delta t=0.272 \mathrm{ps}$ and $\lambda_{0}=76.733 \mathrm{~mm}$. Note the increase in the group velocity compared to Fig. 3, for example. deviations $\left(\zeta_{L}=1 \%, 5 \%, 10 \%\right)$ are considered. For each distribution, an ensemble with 15 realizations is used.

The solid line in Fig. 15 interpolates the (ensemble averaged) peak value of $\left|E_{x}\right|$ inside lossless MPCs for various $\zeta_{L}$. A narrowband incident pulse is employed for these results. As seen from this figure, small deviations in the layer thickness have a strong influence on the MPC response. For instance, in the case of a standard deviation of $\zeta_{L}=5 \%$, the peak amplitude is decreased to about half of that in the optimal MPC.

\section{CONCLUSIONS}

An analysis of transient electromagnetic pulse propagation in spectrally asymmetric MPCs exhibiting ferromagnetic losses has been presented. The analysis is performed by means of a PML-FDTD algorithm based on D-H and B-E formulations that decouples the (anisotropic, dispersive) constitutive equations inside the MPC from the (modified PML) Maxwell equations. In order to analyze frozen modes in MPCs that require long time integrations, frequencydispersive material (bulk) tensors have been integrated in the time-domain update using the ADE approach (since the alternative RC approach was shown to lead to late-time instabilities). Both complex-frequency-shifted PML and standard PML methods have been implemented into FDTD, leading to practically equivalent results in this case, and yielding no instabilities for simulations involving up to $160 \times 10^{6}$ time steps.

A sensitivity analysis was carried out to examine the effect of ferromagnetic losses, frequency mismatch, random $A$ layer misalignment, and random layer thickness on the electromagnetic pulse propagation inside a MPC. As expected, the frozen mode is particularly sensitive to the central frequency of the excitation pulse, with its properties rapidly

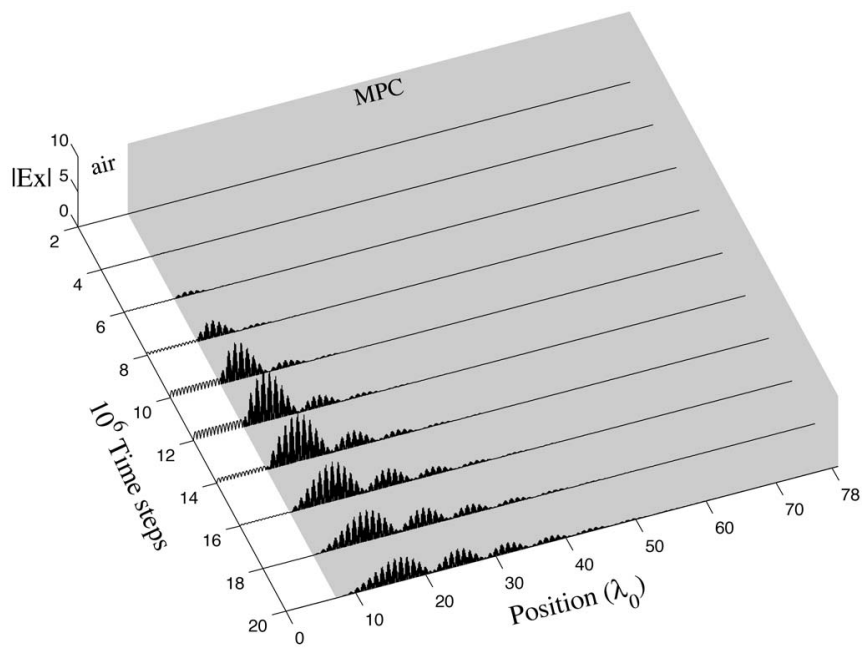

FIG. 12. Snapshots of $\left|E_{x}\right|$ in the presence of a MPC with lossy $\left(\alpha=10^{-4}\right) F$ layers (narrowband incident pulse of unit amplitude). $\Delta t=0.272 \mathrm{ps}, \lambda_{0}=76.733 \mathrm{~mm}$. The effective group velocity is not significantly impacted compared to the lossless MPC in Fig. 3. The $\left|E_{x}\right|$ amplitude is decreased relative to Fig. 3, and this is accentuated as the wave penetrates more deeply into the MPC. 


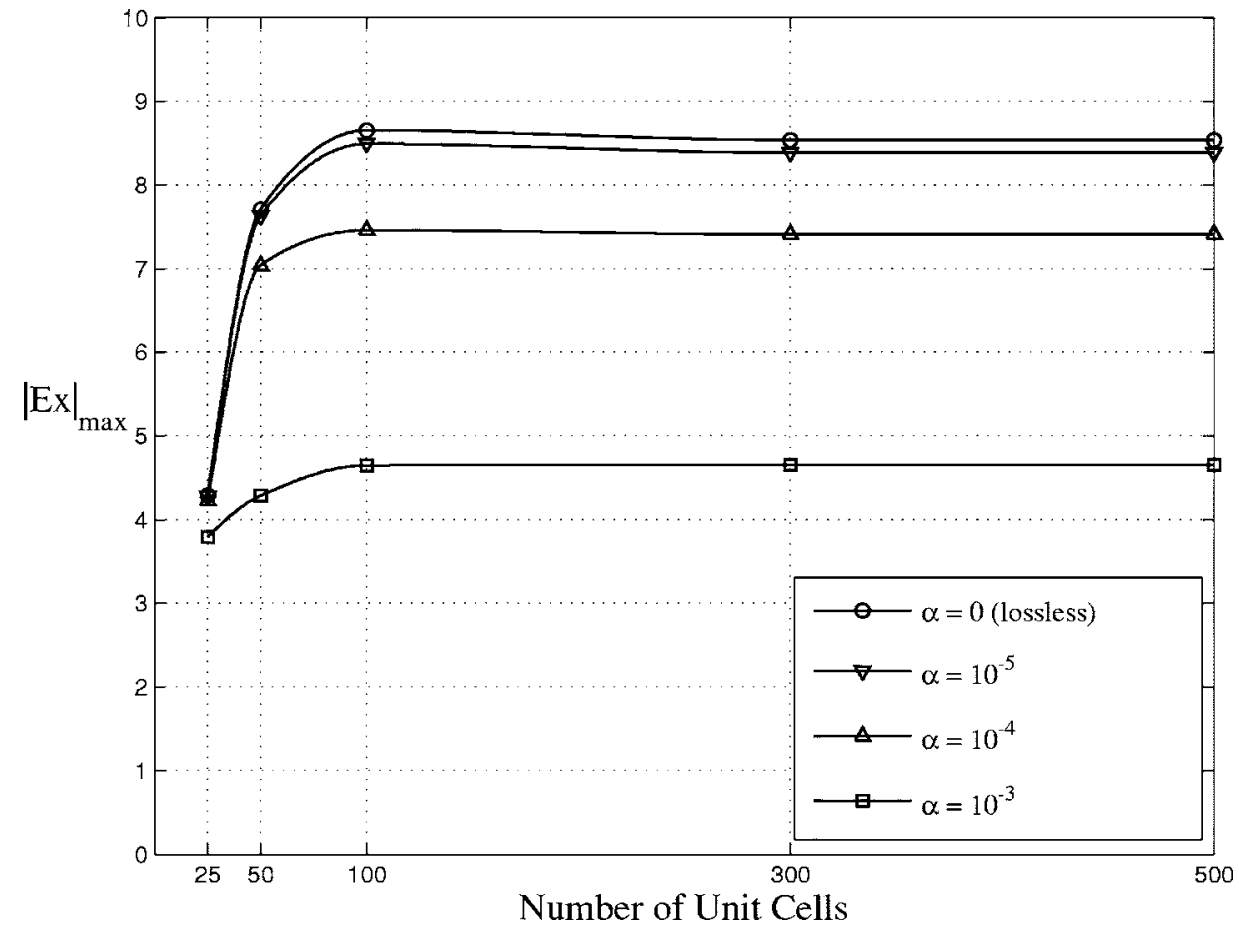

FIG. 13. $\left|E_{x}\right|_{\max }$ inside MPCs with different numbers of unit cells and ferromagnetic loss parameters. Four loss parameters, $\alpha=0$, $10^{-5}, 10^{-4}, 10^{-3}$, and five different numbers of unit cells $(25,50,100,300,500)$ are considered.

deteriorating for frequency-shifted pulses. In the examples considered, inclusion of ferromagnetic loss coefficients equal to $\alpha=10^{-4}$ still yielded frozen modes, and an amplitude increase of about $87 \%$ compared to the optimal (lossless) case, albeit with progressive amplitude deterioration inside the MPC. The peak amplitude also deteriorates when (Gaussiandistributed) small random variations are included in the geometry of the MPC, with a decrease of about a half in the

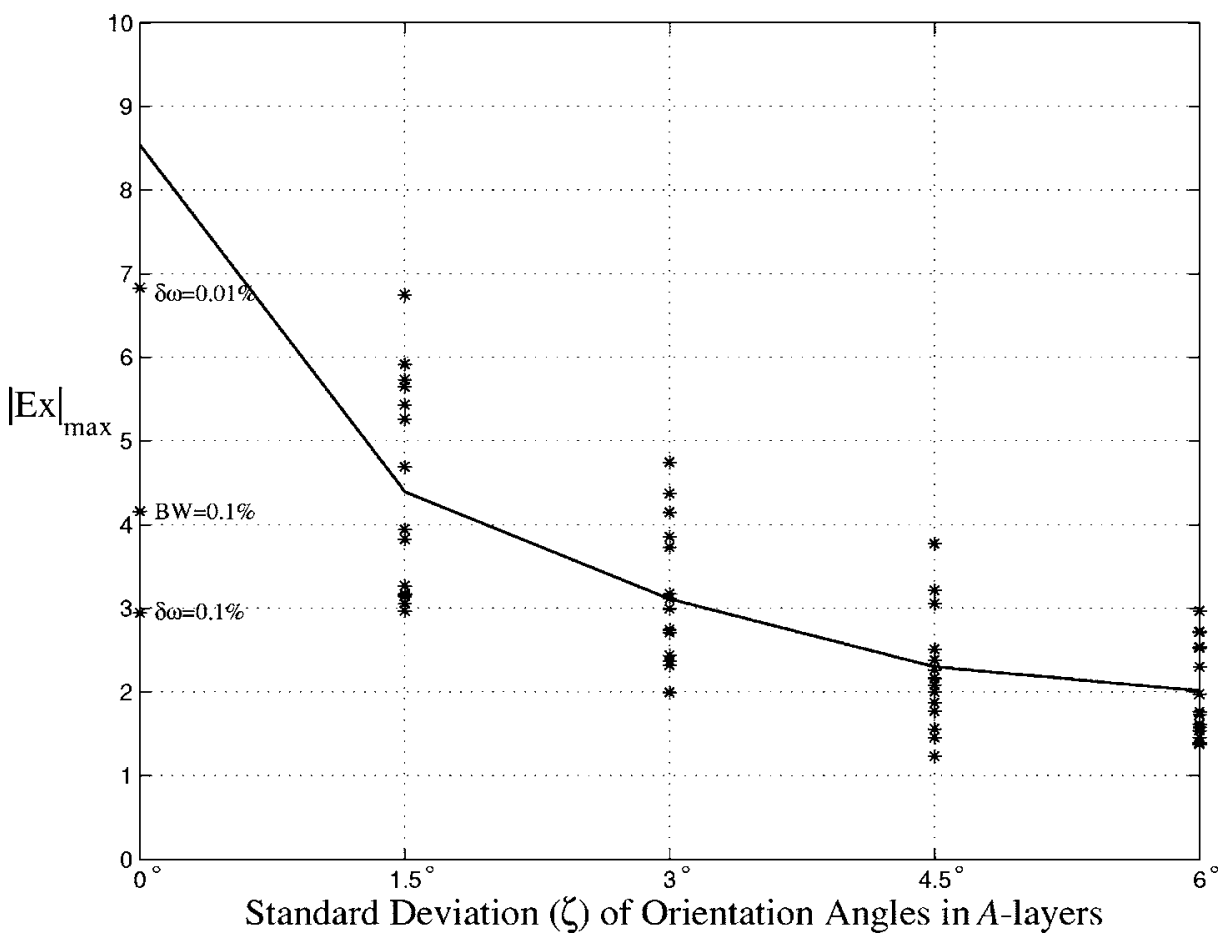

FIG. 14. $\left|E_{x}\right|_{\max }$ inside lossless MPCs with random deviations in the misalignment of $A$ layers. An incidence pulse with BW of $0.01 \%$ is considered. For each standard deviation, 15 realizations are produced, leading to the results indicated by the star symbols. The average results for each standard deviation are interpolated by the solid line. Results for incident pulses with incident pulse with $\delta \omega=0.01 \%$ (for BW $=0.01 \%$ ), $\delta \omega=0.1 \%$ (for $\mathrm{BW}=0.01 \%$ ), and $\mathrm{BW}=0.1 \%$ (for $\delta \omega=0 \%$ ) are also displayed for reference. 


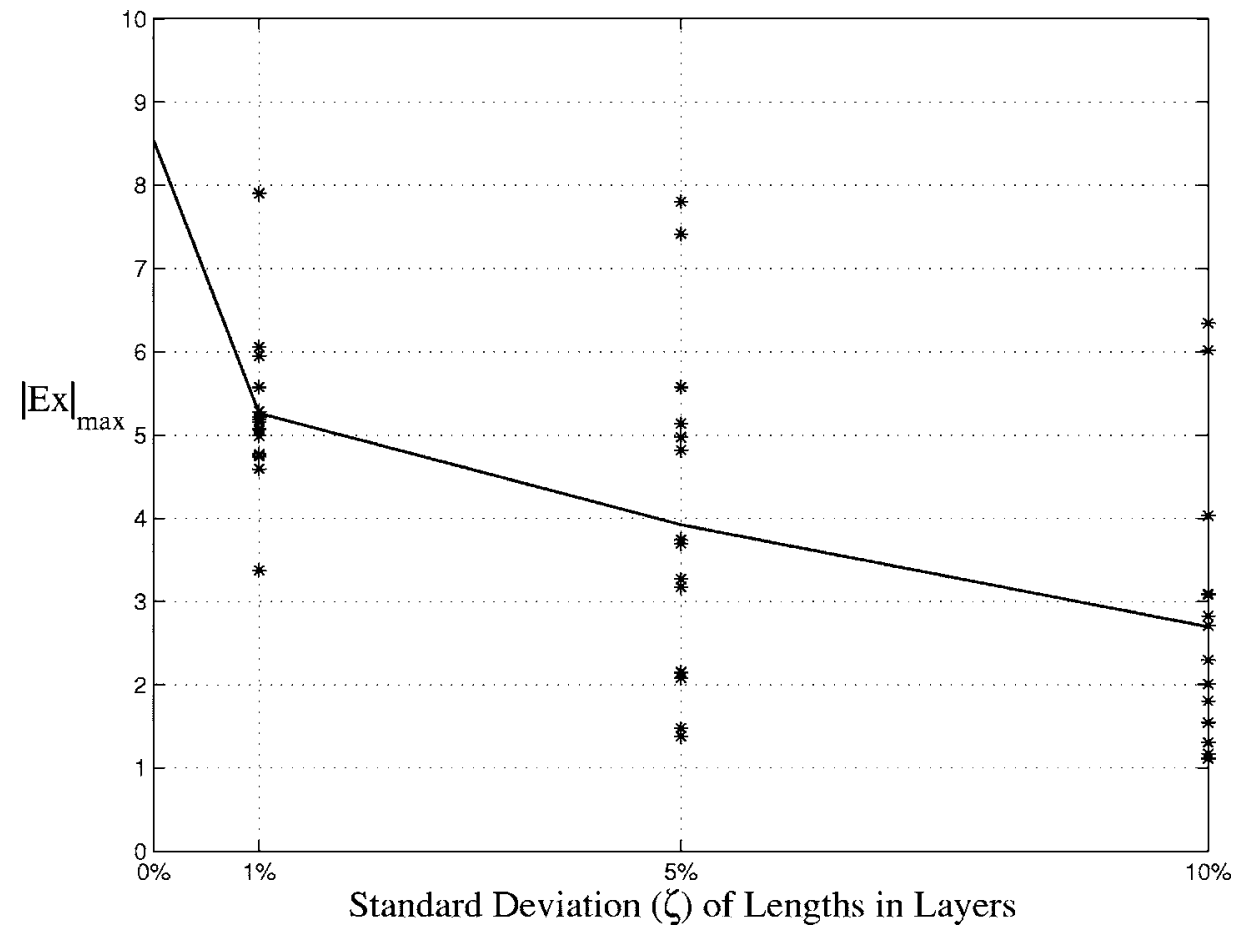

FIG. 15. $\left|E_{x}\right|_{\max }$ inside lossless MPCs with random deviations in the thickness of $A$ and $F$ layers. An incidence pulse with BW of $0.01 \%$ is considered. For each standard deviation utilized for the thickness, 15 realizations are produced, leading to the results indicated by the star symbols. The average results for each standard deviation are interpolated by the solid line.

electric field peak amplitude for a standard deviation of $1.5^{\circ}$ in the relative misalignment of $A$ layers and a standard deviation of 5\% in the thickness of $A$ and $F$ layers, respectively.

\section{ACKNOWLEDGMENTS}

This work has been partially supported by AFOSR under MURI Grant No. FA 9550-04-1-0359, NSF under Grant No. ECS-0347502, and OSC under Grants No. PAS-0061 and No. PAS-0110.

\section{APPENDIX: FDTD FORMULATION}

\section{PML-FDTD update formulation for Maxwell's curl equations}

For arbitrary materials, Maxwell's curl equations can be written as

$$
\begin{gathered}
\frac{\partial \mathbf{D}}{\partial t}=\boldsymbol{\nabla} \times \mathbf{H}, \\
\frac{\partial \mathbf{B}}{\partial t}=-\nabla \times \mathbf{E} .
\end{gathered}
$$

Second-order FDTD update equations for the $\mathbf{D}$ and $\mathbf{B}$ fields in the above can be derived by using central differencing in a standard fashion. Modified finite differences can also be implemented to yield low numerical dispersion error over prescribed frequency ranges. ${ }^{11}$ The PML is incorporated through a modified nabla operator with complex stretching variables: ${ }^{14,15}$

$$
\boldsymbol{\nabla} \rightarrow \tilde{\boldsymbol{\nabla}}=\hat{x} \frac{1}{s_{x}} \frac{\partial}{\partial x}+\hat{y} \frac{1}{s_{y}} \frac{\partial}{\partial y}+\hat{z} \frac{1}{s_{z}} \frac{\partial}{\partial z},
$$

where a complex-frequency-shifted (CFS) stretching ${ }^{22-24}$ is utilized so that

$$
s_{\xi}=\kappa_{\xi}+\frac{\sigma_{\xi}}{\nu_{\xi}+j \omega \epsilon_{0}},
$$

where $\xi=x, y, z$. In the above, $\sigma_{\xi}$ is the conductivity profile along the $\xi$ direction, $\sigma_{\xi} \geq 0$. Moreover, $\kappa_{\xi} \geq 1$ and $\nu_{\xi} \geq 0$ in practice. The above recovers the standard PML stretching ${ }^{14}$ if $\nu_{\xi}=0$. The CFS stretching $\left(\nu_{\xi} \neq 0\right)$ leads to a slightly more costly time-domain implementation than the standard PML stretching $\left(\nu_{\xi}=0\right)$, but the former is more effective at absorbing evanescent waves and low-frequency fields (since it correctly recovers the elliptic equation regime for $\omega \rightarrow 0$ ). Moreover, it does not admit spurious late-time linear growth of axial fields that otherwise may appear in some problems, as pointed out in Ref. 24. Here, we have implemented both the CFS and standard PML stretching functions. We have found that the relative difference between the PML implementations based on these two stretching functions is consistently below $0.1 \%$ and that both implementations yield stable results even for $160 \times 10^{6}$ time steps. The FDTD update for the Maxwell curl equations is derived based on the unsplit PML implementation, by incorporating the ADE approach directly into the PML equations. ${ }^{7}$ 


\section{FDTD update for the constitutive relations}

\section{Dielectric response $D=\overline{\bar{\epsilon}}(\omega) \mathbf{E}(\omega)$}

For $A$ layers [Eq. (1a)], FDTD update equations for $\mathbf{E}$ fields can be easily obtained as

$$
\begin{gathered}
E_{x}^{n}=\frac{\epsilon_{y y}}{\epsilon_{0} \Lambda_{e}} D_{x}^{n}-\frac{\epsilon_{x y}}{\epsilon_{0} \Lambda_{e}} D_{y}^{n}, \\
E_{y}^{n}=\frac{\epsilon_{x x}}{\epsilon_{0} \Lambda_{e}} D_{y}^{n}-\frac{\epsilon_{x y}}{\epsilon_{0} \Lambda_{e}} D_{x}^{n}, \\
E_{z}^{n}=\frac{1}{\epsilon_{0} \epsilon_{z z}} D_{z}^{n},
\end{gathered}
$$

where $\Lambda_{e}=\epsilon_{x x} \epsilon_{y y}-\epsilon_{x y}^{2}$. The superscript $n$ indicates that the field is calculated at $t=n \Delta t$. Note that the $E_{x}$ field depends on both $D_{x}$ and $D_{y}$, and, similarly, the $E_{y}$ depends on both $D_{x}$ and $D_{y}$. The FDTD $\mathbf{E}$ field update equations for the $F$ layers [Eq. (2a)] are trivial, and are not included here.

\section{Ferromagnetic response $B=\overline{\bar{\mu}}(\omega) \mathbf{H}(\omega)$}

For the $F$ layers [Eq. (2b)], $\mathbf{H}$ fields are related to $\mathbf{B}$ fields in the frequency domain as shown in Eq. (A5). From Eq. (2b), the $H_{z}$ field update equation for $F$ layers is straightforward, and hence not included here:

$$
\begin{aligned}
B_{x}(\omega)= & \mu_{0} H_{x}(\omega)+\mu_{0} \frac{\left(\omega_{0}+j \omega \alpha\right) \omega_{m}}{\left(\omega_{0}+j \omega \alpha\right)^{2}+(j \omega)^{2}} H_{x}(\omega) \\
& +\mu_{0} \frac{j \omega \omega_{m}}{\left(\omega_{0}+j \omega \alpha\right)^{2}+(j \omega)^{2}} H_{y}(\omega), \\
B_{y}(\omega)= & \mu_{0} H_{y}(\omega)+\mu_{0} \frac{\left(\omega_{0}+j \omega \alpha\right) \omega_{m}}{\left(\omega_{0}+j \omega \alpha\right)^{2}+(j \omega)^{2}} H_{y}(\omega) \\
& -\mu_{0} \frac{j \omega \omega_{m}}{\left(\omega_{0}+j \omega \alpha\right)^{2}+(j \omega)^{2}} H_{x}(\omega) .
\end{aligned}
$$

After multiplying the denominator on the right-hand side of Eq. (A5) on both sides and using the inverse Fourier transform, we obtain the following equations in the time domain:

$$
\begin{aligned}
(1+ & \left.\alpha^{2}\right) \frac{\partial^{2} B_{x}}{\partial t^{2}}+2 \omega_{0} \alpha \frac{\partial B_{x}}{\partial t}+\omega_{0}^{2} B_{x} \\
= & \mu_{0}\left(1+\alpha^{2}\right) \frac{\partial^{2} H_{x}}{\partial t^{2}}+\mu_{0} \alpha\left(2 \omega_{0}+\omega_{m}\right) \frac{\partial H_{x}}{\partial t} \\
& +\mu_{0} \omega_{0}\left(\omega_{0}+\omega_{m}\right) H_{x}+\mu_{0} \omega_{m} \frac{\partial H_{y}}{\partial t}, \\
(1+ & \left.\alpha^{2}\right) \frac{\partial^{2} B_{y}}{\partial t^{2}}+2 \omega_{0} \alpha \frac{\partial B_{y}}{\partial t}+\omega_{0}^{2} B_{y} \\
= & \mu_{0}\left(1+\alpha^{2}\right) \frac{\partial^{2} H_{y}}{\partial t^{2}}+\mu_{0} \alpha\left(2 \omega_{0}+\omega_{m}\right) \frac{\partial H_{y}}{\partial t} \\
& +\mu_{0} \omega_{0}\left(\omega_{0}+\omega_{m}\right) H_{y}-\mu_{0} \omega_{m} \frac{\partial H_{x}}{\partial t} .
\end{aligned}
$$

Using the central differencing for first- and second-order derivatives, and double averaging over $\Delta t$ for zeroth-order derivatives, we obtain

$$
\begin{aligned}
a_{1} B_{x}^{n+1 / 2}+a_{2} B_{x}^{n-1 / 2}+a_{3} B_{x}^{n-3 / 2}= & b_{1} H_{x}^{n+1 / 2}+b_{2} H_{x}^{n-1 / 2} \\
& +b_{3} H_{x}^{n-3 / 2}+b_{4} H_{y}^{n+1 / 2} \\
& -b_{4} H_{y}^{n-3 / 2}, \\
a_{1} B_{y}^{n+1 / 2}+a_{2} B_{y}^{n-1 / 2}+a_{3} B_{y}^{n-3 / 2}= & b_{1} H_{y}^{n+1 / 2}+b_{2} H_{y}^{n-1 / 2} \\
& +b_{3} H_{y}^{n-3 / 2}-b_{4} H_{x}^{n+1 / 2} \\
& +b_{4} H_{x}^{n-3 / 2},
\end{aligned}
$$

where

$$
\begin{gathered}
a_{1}=\omega_{0}^{2} \Delta t^{2}+4 \omega_{0} \alpha \Delta t+4 \alpha^{2}+4, \\
a_{2}=2 \omega_{0}^{2} \Delta t^{2}-8 \alpha^{2}-8, \\
a_{3}=\omega_{0}^{2} \Delta t^{2}-4 \omega_{0} \alpha \Delta t+4 \alpha^{2}+4, \\
b_{1}=\mu_{0}\left[\omega_{0}\left(\omega_{0}+\omega_{m}\right) \Delta t^{2}+2 \alpha\left(2 \omega_{0}+\omega_{m}\right) \Delta t+4 \alpha^{2}+4\right], \\
b_{2}=\mu_{0}\left[2 \omega_{0}\left(\omega_{0}+\omega_{m}\right) \Delta t^{2}-8 \alpha^{2}-8\right], \\
b_{3}=\mu_{0}\left[\omega_{0}\left(\omega_{0}+\omega_{m}\right) \Delta t^{2}-2 \alpha\left(2 \omega_{0}+\omega_{m}\right) \Delta t+4 \alpha^{2}+4\right], \\
b_{4}=2 \mu_{0} \omega_{m} \Delta t .
\end{gathered}
$$

By solving Eqs. (A7a) and (A7b) simultaneously, FDTD update equations for $H_{x}$ and $H_{y}$ can be finally obtained:

$$
\begin{aligned}
H_{x}^{n+1 / 2}= & -\frac{b_{1} b_{2}}{b_{1}^{2}+b_{4}^{2}} H_{x}^{n-1 / 2}-\frac{b_{1} b_{3}-b_{4}^{2}}{b_{1}^{2}+b_{4}^{2}} H_{x}^{n-3 / 2}+\frac{b_{2} b_{4}}{b_{1}^{2}+b_{4}^{2}} H_{y}^{n-1 / 2} \\
& +\frac{b_{1} b_{4}+b_{3} b_{4}}{b_{1}^{2}+b_{4}^{2}} H_{y}^{n-3 / 2}+\frac{b_{1} a_{1}}{b_{1}^{2}+b_{4}^{2}} B_{x}^{n+1 / 2}+\frac{b_{1} a_{2}}{b_{1}^{2}+b_{4}^{2}} B_{x}^{n-1 / 2} \\
& +\frac{b_{1} a_{3}}{b_{1}^{2}+b_{4}^{2}} B_{x}^{n-3 / 2}-\frac{b_{4} a_{1}}{b_{1}^{2}+b_{4}^{2}} B_{y}^{n+1 / 2}-\frac{b_{4} a_{2}}{b_{1}^{2}+b_{4}^{2}} B_{y}^{n-1 / 2} \\
& -\frac{b_{4} a_{3}}{b_{1}^{2}+b_{4}^{2}} B_{y}^{n-3 / 2}, \\
H_{y}^{n+1 / 2}= & -\frac{b_{1} b_{2}}{b_{1}^{2}+b_{4}^{2}} H_{y}^{n-1 / 2}-\frac{b_{1} b_{3}-b_{4}^{2}}{b_{1}^{2}+b_{4}^{2}} H_{y}^{n-3 / 2}-\frac{b_{2} b_{4}}{b_{1}^{2}+b_{4}^{2}} H_{x}^{n-1 / 2} \\
& -\frac{b_{1} b_{4}+b_{3} b_{4}}{b_{1}^{2}+b_{4}^{2}} H_{x}^{n-3 / 2}+\frac{b_{1} a_{1}}{b_{1}^{2}+b_{4}^{2}} B_{y}^{n+1 / 2}+\frac{b_{1} a_{2}}{b_{1}^{2}+b_{4}^{2}} B_{y}^{n-1 / 2} \\
& +\frac{b_{1} a_{3}}{b_{1}^{2}+b_{4}^{2}} B_{y}^{n-3 / 2}+\frac{b_{4} a_{1}}{b_{1}^{2}+b_{4}^{2}} B_{x}^{n+1 / 2}+\frac{b_{4} a_{2}}{b_{1}^{2}+b_{4}^{2}} B_{x}^{n-1 / 2} \\
& +\frac{b_{4} a_{3}}{b_{1}^{2}+b_{4}^{2}} B_{x}^{n-3 / 2} .
\end{aligned}
$$

Note that it is possible to derive alternative $\mathbf{H}$ field FDTD update equations for ferrite media. For example, the $H_{x}$ and 
$H_{y}$ fields can be first decoupled in the frequency domain (unlike the above), by solving Eqs. (A5a) and (A5b) simultaneously, and later inverse Fourier transformed. However, it was found that the resulting FDTD formulation suffers from late-time instabilities.
If the dc biasing magnetic field is $-z$ oriented, the signs of off-diagonal terms should be changed in Eq. (3b). The corresponding FDTD update equations for $\mathbf{H}$ fields can be simply obtained by changing the signs of coefficients in the off-diagonal terms as

$$
\begin{aligned}
H_{x}^{n+1 / 2}= & -\frac{b_{1} b_{2}}{b_{1}^{2}+b_{4}^{2}} H_{x}^{n-1 / 2}-\frac{b_{1} b_{3}-b_{4}^{2}}{b_{1}^{2}+b_{4}^{2}} H_{x}^{n-3 / 2}-\frac{b_{2} b_{4}}{b_{1}^{2}+b_{4}^{2}} H_{y}^{n-1 / 2}-\frac{b_{1} b_{4}+b_{3} b_{4}}{b_{1}^{2}+b_{4}^{2}} H_{y}^{n-3 / 2}+\frac{b_{1} a_{1}}{b_{1}^{2}+b_{4}^{2}} B_{x}^{n+1 / 2}+\frac{b_{1} a_{2}}{b_{1}^{2}+b_{4}^{2}} B_{x}^{n-1 / 2}+\frac{b_{1} a_{3}}{b_{1}^{2}+b_{4}^{2}} B_{x}^{n-3 / 2} \\
& +\frac{b_{4} a_{1}}{b_{1}^{2}+b_{4}^{2}} B_{y}^{n+1 / 2}+\frac{b_{4} a_{2}}{b_{1}^{2}+b_{4}^{2}} B_{y}^{n-1 / 2}+\frac{b_{4} a_{3}}{b_{1}^{2}+b_{4}^{2}} B_{y}^{n-3 / 2}, \\
H_{y}^{n+1 / 2}= & -\frac{b_{1} b_{2}}{b_{1}^{2}+b_{4}^{2}} H_{y}^{n-1 / 2}-\frac{b_{1} b_{3}-b_{4}^{2}}{b_{1}^{2}+b_{4}^{2}} H_{y}^{n-3 / 2}+\frac{b_{2} b_{4}}{b_{1}^{2}+b_{4}^{2}} H_{x}^{n-1 / 2}+\frac{b_{1} b_{4}+b_{3} b_{4}}{b_{1}^{2}+b_{4}^{2}} H_{x}^{n-3 / 2}+\frac{b_{1} a_{1}}{b_{1}^{2}+b_{4}^{2}} B_{y}^{n+1 / 2}+\frac{b_{1} a_{2}}{b_{1}^{2}+b_{4}^{2}} B_{y}^{n-1 / 2}+\frac{b_{1} a_{3}}{b_{1}^{2}+b_{4}^{2}} B_{y}^{n-3 / 2} \\
& -\frac{b_{4} a_{1}}{b_{1}^{2}+b_{4}^{2}} B_{x}^{n+1 / 2}-\frac{b_{4} a_{2}}{b_{1}^{2}+b_{4}^{2}} B_{x}^{n-1 / 2}-\frac{b_{4} a_{3}}{b_{1}^{2}+b_{4}^{2}} B_{x}^{n-3 / 2} .
\end{aligned}
$$

${ }^{1}$ A. Figotin and I. Vitebsky, Phys. Rev. E 63, 066609 (2001).

${ }^{2}$ A. Figotin and I. Vitebskiy, Phys. Rev. B 67, 165210 (2003).

${ }^{3}$ A. Figotin and I. Vitebskiy, in Proceedings of the IEEE Antennas and Propagation International Symposium, Monterey, CA, 2004 (IEEE, New York, 2004), pp. 3789-3791.

${ }^{4}$ G. Mumcu, K. Sertel, J. L. Volakis, I. Vitebskiy, and A. Figotin, in Proceedings of the IEEE Antennas and Propagation International Symposium, Monterey, CA, 2004 (Ref. 3), pp. 1395-1398.

${ }^{5}$ R. M. Joseph, S. C. Hagness, and A. Taflove, Opt. Lett. 16, 1412 (1991).

${ }^{6}$ O. P. Gandhi, B.-Q. Gao, and J.-Y. Chen, IEEE Trans. Microwave Theory Tech. 41, 658 (1993).

${ }^{7}$ F. L. Teixeira and W. C. Chew, IEEE Trans. Geosci. Remote Sens. 38, 1530 (2000).

${ }^{8}$ H.-T. Chien, H.-T. Tang, C.-H. Kuo, C.-C. Chen, and Z. Ye, Phys. Rev. B 70, 113101 (2004).

${ }^{9}$ A. Vial, A.-S. Grimault, D. Macias, D. Barchiesi, and M. L. de la Chapelle, Phys. Rev. B 71, 085416 (2005).

${ }^{10}$ L. San Martin and Y. Oono, Phys. Rev. E 57, 4795 (1998).

${ }^{11}$ S. Wang and F. L. Teixeira, Phys. Rev. E 69, 016701 (2004).

${ }^{12}$ A. P. Zhao, IEEE Trans. Microwave Theory Tech. 46, 1511 (1998).

${ }^{13}$ O. Ramadan and A. Y. Oztoprak, IEEE Microw. Wirel. Compon. Lett. 13, 402 (2003).
${ }^{14}$ W. C. Chew and W. H. Weedon, Microwave Opt. Technol. Lett. 7, 599 (1994).

${ }^{15}$ F. L. Teixeira and W. C. Chew, IEEE Microw. Guid. Wave Lett. 7, 371 (1997).

${ }^{16}$ J. A. Pereda, L. A. Vielva, A. Vegas, and A. Prieto, IEEE Microw. Guid. Wave Lett. 3, 136 (1993).

${ }^{17}$ K. S. Kunz and R. J. Luebbers, The Finite Difference Time Domain Method for Electromagnetics (CRC Press, Boca Raton, FL, 1993).

${ }^{18}$ J. W. Schuster and R. J. Luebbers, Radio Sci. 31, 923 (1996).

${ }^{19}$ A. Taflove and S. C. Hagness, Computational Electrodynamics: The Finite-Difference Time-Domain Method, 2nd ed. (Artech House, Norwood, MA, 2000).

${ }^{20}$ D. M. Pozar, Microwave Engineering, 3rd ed. (John Wiley \& Sons, New York, 2004)

${ }^{21}$ R. W. Ziolkowski and E. Heyman, Phys. Rev. E 64, 056625 (2001).

${ }^{22}$ M. Kuzuoglu and R. Mittra, IEEE Microw. Guid. Wave Lett. 6, 447 (1996).

${ }^{23}$ J. A. Roden and S. D. Gedney, Microwave Opt. Technol. Lett. 27, 334 (2000).

${ }^{24}$ E. Becache, P. G. Petropoulos, and S. D. Gedney, IEEE Trans. Antennas Propag. 52, 1335 (2004). 